\title{
Rapid diagnostic assays in the genomic biology era: detection and identification of infectious disease and biological weapon agents
}

\author{
Leonard F. Peruski, Jr. and Anne Harwood Peruski
}

BioTechniques 35:840-846 (October 2003)

\begin{abstract}
In this special section of BioTechniques, we examine the role of rapid molecular technologies in the detection and identification of agents of infectious disease (ID) and biological weapons (BWs). Besides the threat posed by the global proliferation of BW technologies, there are numerous emerging and reemerging ID agents with significant public health consequences. Further compounding this already complicated situation are the estimated 600 million international tourists annually, many with the potential to the spread disease globally in a matter of hours. While clinical laboratories have key roles in the detection and identification of potential ID/BW agents, most staff are unfamiliar with these agents because of their rarity and the often laborious conventional methodologies needed to identify them. To meet this challenge, a vast array of rapid assay strategies has been developed for use in clinical diagnostics and environmental detection. Technologies have been developed or adapted to the challenges posed by these agents, permitting detection and identification in several minutes to hours. In particular, the development of improved reagents and detection systems has led to dramatic improvements in the sensitivity and specificity of immunological and nucleic acid-based systems, allowing an ever-increasing range of analytes to be identified and quantitated. In the accompanying articles, we have brought together experts from the many overlapping aspects of this arena in order to present a comprehensive and critical analysis of these technologies.
\end{abstract}

\section{NATURE OF THE PROBLEM}

\section{Biological Weapon Agents}

The global proliferation of biological weapon (BW) technologies remains a significant threat to public health and the environment. Unfortunately, many BW agents (Table 1) are unfamiliar to most clinicians and laboratory scientists, complicating appropriate responses.

Key attributes of a "good" BW agent are aerosolization, infectivity or toxicity, and disease severity (1). In addition, criteria such as access to the potential agent, simplicity of production and stability, coupled with communicability and ease of dispersal, determine which prospective agents are the most likely to be utilized. For maximum effect, an optimal agent should result in significant morbidity and/or mortality, be simple to manufacture, and have limited options for preventive or prophylactic treatment. As the respiratory route is considered the most effective for dispersal, stability in an aerosol form (particle size approximately $1-10 \mu \mathrm{m}$ ) and the capability to be disseminated in this manner are key. Based on these criteria, Bacillus anthracis (anthrax) and variola major virus (smallpox) are considered to have the greatest potential for mass casualties and civil disrup- tion (1-4). Also high on a prospective list of agents are the neurotoxins of Clostridium botulinum (botulism), Yersinia pestis (bubonic and pneumonic plague), and Francisella tularensis (rabbit fever, undulant fever) (1-4). Lower on the list are Burkholderia pseudomallei and mallei, Rickettsia species, Coxiella burnetti, Venezuelan equine encephalitis virus, Marburg and Ebola viruses, and influenza viruses (1-4).

\section{Emerging Infectious Disease Agents}

In addition to diseases caused by intentional epidemics, there are several emerging infectious diseases (IDs) with the potential for significant public health consequences, including cholera, dengue fever, malaria, Rift Valley fever, severe acute respiratory syndrome (SARS), and West Nile fever (Table 1) (1-4). While the interaction of several complex factors influence the emergence of ID, including genetic variation in the host and pathogen, environmental changes, and population pressures (2), compounding this already complicated situation are the over 600 million tourists per year that travel internationally. As demonstrated by the SARS epidemic in spring 2003, many of these individuals have the potential to the spread disease globally in mere hours. 
Table 1. Potential Biological Weapon and Infectious Disease Threat Agents

\begin{tabular}{|ll|}
\hline Bacteria & Toxins \\
Bacillus anthracis & Aflatoxins \\
Brucella species & Clostridium botulinum neurotoxins \\
Burkholderia species & Ricin \\
Coxiella burnetii & Saxitoxin \\
Escherichia coli O157:H7 & Shigatoxin \\
Francisella tularensis & Staphylococcal enterotoxins \\
Salmonella species & \\
Shigella species & Viruses \\
Vibrio cholerae & Dengue fever \\
Yersinia pestis & Ebola \\
& Rift Valley fever \\
Parasites & SARS \\
Plasmodium species (malaria) & Smallpox \\
Cryptosporidium parvum & West Nile fever \\
& Yellow fever \\
\hline
\end{tabular}

\section{LABORATORY METHODS}

\section{Overview}

Because of the threat posed by both BW and emerging ID agents, there is a need to rapidly identify such agents in order to treat individuals at risk and limit disease transmission, improve public health surveillance and epidemiology, and monitor environmental impact. As shown by the anthrax attack during the fall of 2001 and the SARS outbreak of 2002-2003, patients would begin appearing at hospitals and clinics within several days of exposure, most presenting with nonspecific flu-like symptoms (5-7). The first days of the outbreak might not even cause undue concern. Confusion with endemic IDs well may delay the application of appropriate therapies and prophylactic measures. Unfortunately, for many infections, the prognosis of the patient will be poor once the distinguishing clinical signs are recognized. Similar issues and problems would result from environmental or agricultural contamination by the intentional release of BW/ID agents, although the time available to plan and initiate a response to an event may be longer.

As a result, medical-care providers and laboratory workers must be prepared to recognize unusual events, uncommon agents, and unexplained illnesses. However, the usefulness of current culture and identification methods as a part of the surveillance process is limited by the time required to obtain a satisfactory answer and by the sensitivity and specificity of the selected approach. In addition, some microorganisms are difficult or impossible to culture under routine conditions in the laboratory. Because of the variable and substantial delay that may occur after exposure and the onset of disease, rapid and accurate identification of biological threats is necessary to protect public health and safety $(6-8)$.

To meet this challenge, a vast array of assay strategies have been developed over the past 25 years for clinical and environmental detection, which, in many instances, have reduced detection and identification to a few hours or even several minutes. In particular, the development of improved reagents and detection equipment has led to dramatic improvements in the sensitivity and specificity of such assays, allowing an ever-increasing range of agents to be identified and quantitated.

\section{Conventional Methods}

Most conventional or traditional microbiological approaches for identifying biological agents work best when high concentrations of the agent are present and, in the case of clinical disease, when the patient is already critically ill $(9,10)$. Even with those caveats, the most sensitive and widely used method for identifying the broadest range of viable agents remains culture and other related methods (Table 2). There are two key reasons for this continued supremacy. First, they can detect many agents at sublethal levels (11). Second, the capability to do basic culture, staining, and microscopy exist throughout the public health laboratory system and at most universities $(9,12,13)$, and as they do not require a substantial investment in new technology or equipment, they can be rapidly implemented in a broad range of public and private laboratories $(12,13)$.

However, there are significant drawbacks to reliance solely on these methodologies. Most laboratory personnel require additional training and certification to accurately identify uncommon agents. Some laboratories continue to lack infrastructure, critical equipment, and diagnostic reagents to identify unusual organisms. In particular, proper biological safety level (BSL)-2 and BSL-3 facilities remain in short supply, as do the trained personnel necessary to operate them (8). Overall, traditional methods tend to be the most labor- and resource-intensive and often require considerable expertise. Moreover, suspect isolates often must be further characterized by molecular techniques to confirm identification (10).

\section{Nucleic Acid Methods}

As reviewed in the accompanying article by Ivnitski and co-authors (14), nucleic acid-based techniques have enormous potential in the detection and identification of BW/ID agents because of their specificity, sensitivity, and the speed with which results can be obtained (15). Gene amplification, or $\mathrm{PCR}$, is a sensitive approach for agent identification and the preferred method for identifying nonviable (or inactivated) organisms (Table 2). The identification of ID agents by PCR methods is now routine for many large clinical laboratories and reference centers (16). Several gene amplification assays for the key BW/ID agents have been described (Table 3).

Of particular note is the revolutionary role that "real-time" or quantitative PCR has played in the detection of BW/ID agents, especially in the recent outbreaks of anthrax and SARS (17-21). Reaction chemistries such as probe-hydrolysis, typified by the TaqMan ${ }^{\circledR}$ process (Applied Biosystems, Foster City, CA, USA), have made real-time detection practical (22-24). Commercial quantitative PCR systems are available from several sources, including Applied Biosystems, 
Table 2. Relative Limits of Detection of Selected Identification Technologies for Bacillus anthracis

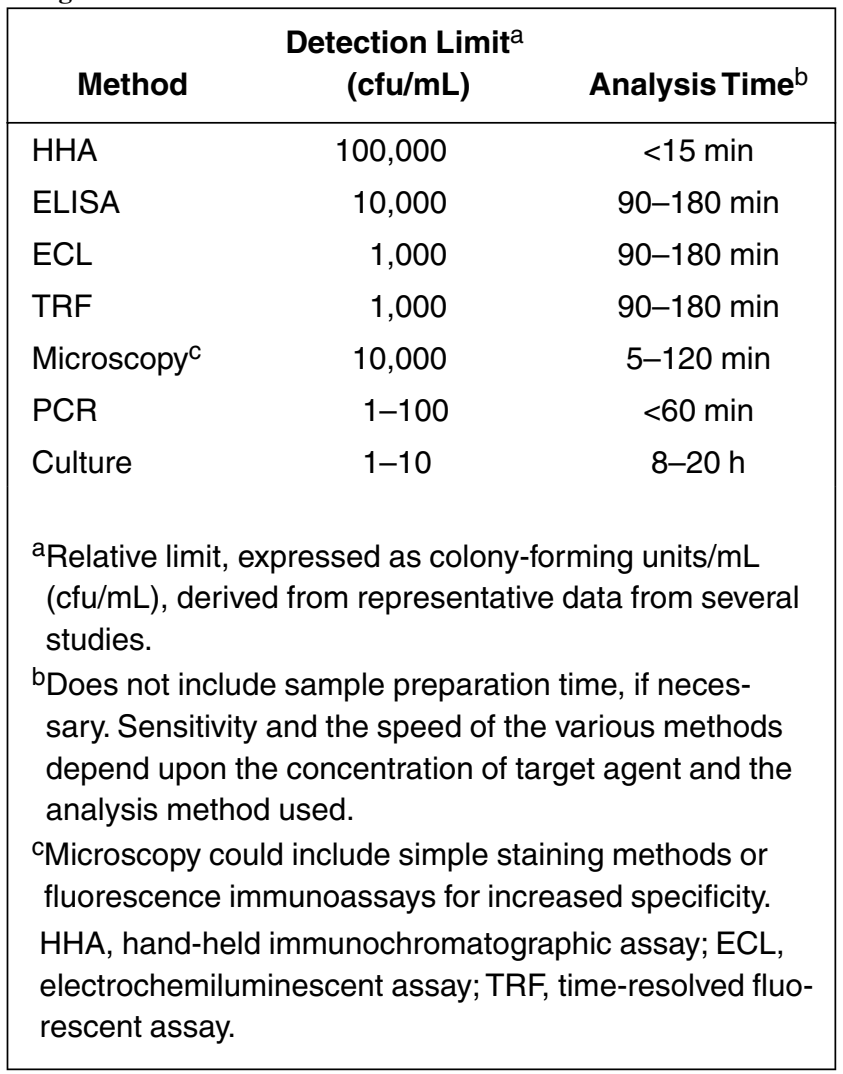

Bio-Rad (Hercules, CA, USA), Idaho Technologies (Salt Lake City, UT, USA), Cepheid (Sunnyvale, CA, USA), and Roche Diagnostics (Indianapolis, IN, USA). Over 50 quantitative PCR assays against 26 infectious agents have been developed based on this reaction chemistry by scientists in the Department of Defense, the Centers for Disease Control (CDC), and the Department of Energy (25). Using new sample processing techniques, the presumptive identification of most biological agents can be completed in less than 1 hour with quantitative PCR methods, compared with approximately 6 hours with older PCR methods and days with culture-based methods.

Microarrays of nucleic acids, popularly referred to as "gene chips", are an exciting recent development with potential applications ranging from pathogen subtyping to the identification of unknown microorganisms, while retaining the speed and sensitivity of PCR-based methods. While currently limited to use in applications for identifying genes involved in pathogenicity and analysis of host response to infection, hybridization-based approaches will be extended to the detection of BW/ID agents in the near future (26-28).

There are, however, still significant barriers that must be overcome before such nucleic acid-based methods can be routinely adopted (16). First, such methodologies are often expensive, labor-intensive, and require highly trained personnel. Second, most of these assays perform best with purified substrates, which may take from 1 to 6 hours to prepare depending upon the method used. Often, this processing is unnecessary, however. While using unprocessed samples may lose some sensitivity, the rapidity of analysis and reduced risk of contamination often outweigh the advantages of purified nucleic acids.

\section{Immunological Methods}

In the second accompanying article of this special section, Andreotti and co-authors examine the considerable depth of immunological detection methods (29). Of the methods that they discuss, four are widely used for the detection and identification of potential BW/ID agents: (i) immunochromatographic lateral flow assays ("hand-held" assays or HHA); (ii) ELISA; (iii) time-resolved fluorescence assays (TRF); and (iv) electrochemiluminescence assays (ECL). Examples of other methods appropriate for evaluating clinical specimens include immunofluorescence assays (IFA) and immunohistochemistry (IHC).

While immunoassays are regularly used to confirm a clinical diagnosis (10), they are usually less sensitive and specific for directly detecting most BW/ID agents than culture and gene amplification approaches (Table 2). Without concentrating the specimen prior to analysis, many immunoassays have a sensitivity threshold 100- to 1000-fold above the estimated infectious dose (Table 2), potentially limiting their utility $(6,7)$. Additionally, immunoassays may lack the necessary specificity to be useful for detection, let alone definitive identification. For example, Bacillus species related to B. anthracis, known as the $B$. cereus group, are common contaminants of the environment and share many antigens on the spore surface. As a result, many immunoassays may have specificity problems when used inappropriately with environmental samples. However, while nearly all immunoassays require confirmation using other methods, ELISA, TRF, and ECL, along with IHC and IFA, are effective tools for quickly screening large numbers of samples.

The single most critical reagent for all immunological assays remains the antibody, as the range and scope of immunoassays has largely arisen through the exquisite specificity of different types of antibodies. Over the last quarter century, antibodies have rapidly evolved: polyclonal antibodies have been largely supplanted, by monoclonal derivatives, with recombinantly engineered types potentially poised as the next generation of reagents. As a result, antibody affinity and specificity are the limiting factors of these assays and those selected for use in an assay must be evaluated carefully. Key among the criteria for evaluation is assessment of the ability of the antibody to bind to a desired target with high affinity, while at the same time retaining high specificity. This is determined empirically based on extensive testing with a robust panel of target agents, molecular and environmental mimics, and potential interferents. Such a specificity panel usually includes (or should include) genetic "near-neighbors" as well as material or agents from environmental or biological sources likely to be contaminants of samples.

HHAs are the simplest of these assays to use, taking approximately 15 minutes to run and requiring only the dilution of the test agent in a sample buffer and applying several drops (approximately $200 \mu \mathrm{L}$ ) to the test strip. However, they have significant limitations. Usually only one agent can be detected per assay strip. Thus, if an unknown sample needs to be 
Table 3. Examples of BW/ID Agents, Accepted Detection and Identification Methods, and Available Rapid Assays

\begin{tabular}{|c|c|c|c|c|c|c|}
\hline \multirow[b]{2}{*}{ Agent } & \multicolumn{6}{|c|}{ Detection Methods } \\
\hline & Standards & HHA & ELISA & ECL & TRF & PCR \\
\hline \multicolumn{7}{|l|}{ Bacteria } \\
\hline Bacillus anthracis & Culture, $\gamma$-phage, IFA & $\mathrm{X}$ & $\mathrm{X}$ & $\mathrm{X}$ & $\mathrm{X}$ & $\mathrm{X}$ \\
\hline Brucella species & Culture, IFA & $x$ & $x$ & & & $x$ \\
\hline Burkholderia species & Culture & $\mathrm{X}$ & $\mathrm{x}$ & & & $x$ \\
\hline Clostridium botulinum & Bioassay & $x$ & $x$ & & & $x$ \\
\hline Coxiella burnetii & Culture, serology, IFA & $x$ & $x$ & & & $x$ \\
\hline Escherichia coli O157:H7 & Culture, serology & $x$ & $x$ & $x$ & & $x$ \\
\hline Francisella tularensis & Culture, IFA & $x$ & $\mathrm{X}$ & & $x$ & $x$ \\
\hline Salmonella species & Culture, serology & $x$ & $x$ & & & $x$ \\
\hline Shigella species & Culture & $\mathrm{X}$ & $\mathrm{X}$ & & & $\mathrm{X}$ \\
\hline Vibrio cholerae & Culture, serology & $x$ & $x$ & & & $x$ \\
\hline Yersinia pestis & Culture, IFA & $x$ & $x$ & & & $x$ \\
\hline \multicolumn{7}{|l|}{ Parasites } \\
\hline Plasmodium species & Microscopy & $x$ & $x$ & & & $x$ \\
\hline Cryptosporidium parvum & Microscopy & & $x$ & $x$ & & $x$ \\
\hline \multicolumn{7}{|l|}{ Toxins } \\
\hline Aflatoxins & ELISA, HPLC, MS & $x$ & $x$ & & & \\
\hline C. botulinum neurotoxins & Bioassay & $x$ & $x$ & $x$ & $x$ & \\
\hline Ricin & ELISA & $x$ & $x$ & $x$ & & \\
\hline Saxitoxin & Bioassay & & $x$ & & & \\
\hline Shigatoxin & Bioassay & $x$ & $x$ & & & \\
\hline Staphylococcal enterotoxins & ELISA & $x$ & $x$ & $x$ & $x$ & \\
\hline \multicolumn{7}{|l|}{ Viruses } \\
\hline Dengue & Culture, CF, IFA & $x$ & $x$ & & & $x$ \\
\hline Ebola & Culture, EM, IFA & $x$ & & & $x$ & \\
\hline Rift Valley fever & Culture, IFA & & $x$ & & & $x$ \\
\hline SARS & Culture? & & $x$ & & & $x$ \\
\hline Variola major (smallpox) & Culture, EM & $x$ & $x$ & & & $x$ \\
\hline West Nile fever & Culture, serology & & $x$ & & & $x$ \\
\hline Yellow fever & Culture, CF, IFA & & $x$ & & & $x$ \\
\hline
\end{tabular}

characterized, several assays must usually be run to obtain a presumptive identification. They also tend to be the least sensitive and the least specific of all immunological assays (30), making them useful only as a screening tool. Another key problem is that in the presence of a large excess of antigen, HHAs are subject to the "hook effect," which can lead to false negative test results. These disadvantages are being partially offset by technological advances that improve sensitivity by at least an order of magnitude and make detection quantitative, not merely subjective (31-34).
ELISA, ECL, and TRF are similar "sandwich-type" immunological assays. ELISA, the most widely used and understood of these formats, uses a colorimetric signal that can be directly assessed visually or quantitatively measured by optical density. Because of their combined simplicity and sensitivity, they can be used reliably for screening large numbers of small volume test samples in the simplest of laboratory environments. While the greatest impact of these assays has been in epidemiology and the diagnosis of IDs (35-37), their potential for high-throughput automation makes them 


\section{OVERVIEW}

Table 4. Numeric Measures of Assay Effectiveness

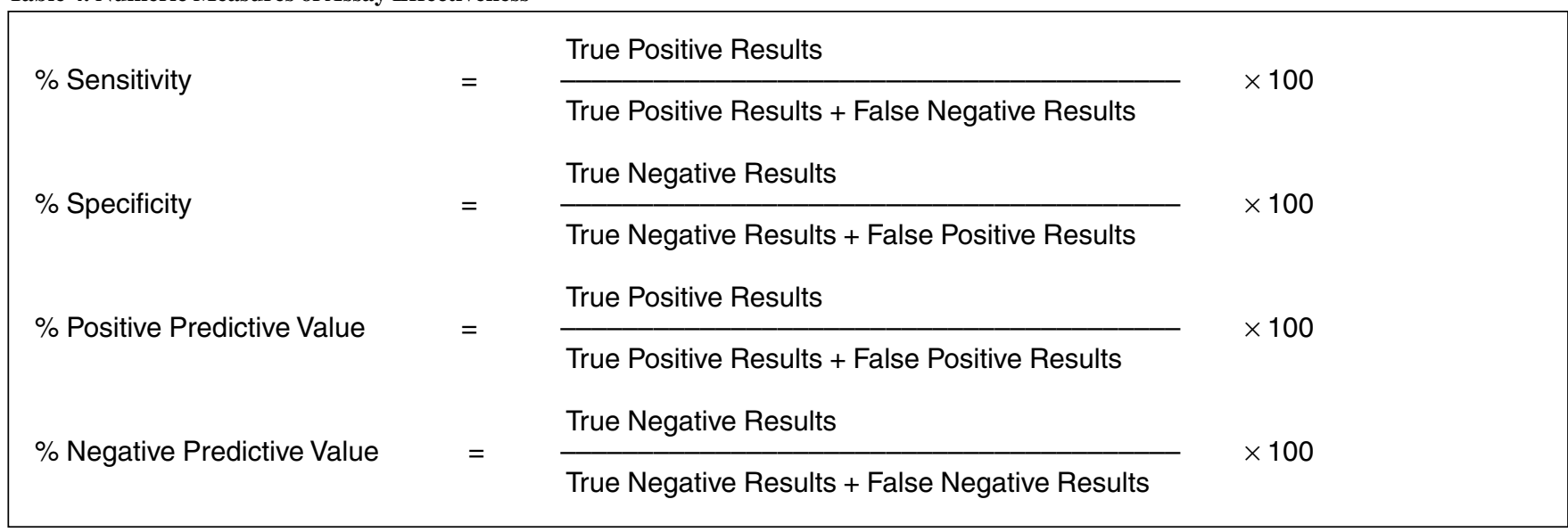

valuable assets in giving laboratories additional "surge capacity" for emergencies.

ECL assays are similar to ELISA, except the detector antibody is directly labeled with a chemiluminescent ligand such as ruthenium, while in TRF assays, the detector antibody is directly labeled with a lanthanide chelate $(30,38,39)$. ECL and TRF assays are more sensitive than ELISA (by up to one order of magnitude), but offsetting this advantage is the need to avoid background contamination, often necessitating the use of dedicated measuring devices and rigorous washing techniques. ECL and TRF assays have similar limitations to ELISA. Primarily, these are a direct function of the antibodies used-more sensitive and specific assays require antibodies of higher avidity and affinity. 


\section{ISSUES IN ASSAY DEVELOPMENT AND VALIDATION}

Introduction of rapid molecular diagnostic methods into a laboratory setting is subject to practical constraints (40). The performance of these methods must be evaluated against accepted standard methods for validation. Signal-to-noise ratios need to be closely examined, and the limit of detection needs to be carefully analyzed $(30,38,41)$. Once in the laboratory setting, rapid assays must be continually monitored for false positive and false negative results.

There are two broadly interrelated metrics for assessing an assay: (i) quantitative metrics and (ii) qualitative metrics. The key quantitative (or numeric) assessments of the effectiveness of any assay are sensitivity, specificity, and predictive value (Table 4). The latter metric is usually expressed as positive predictive value and negative predictive value. Obviously, the more sensitive and specific an assay, the better the assay. Likewise, the higher the positive predictive value and negative predictive value, the better the assay. In addition, several qualitative assessments impact on the utility of an assay, including ease of operation, the training required, sensitivity to contaminants and interferents, and the range of sample types that can be analyzed.

\section{FUTURE TRENDS}

Molecular methods have been used increasingly over the past 20 years to improve the sensitivity and rapidity of detection for BW/ID agents. Despite the considerable advances in such assays, however, the optimal approach for the detection and identification of BW/ID agents remains an integration of conventional and molecular methods (42). Conventional methods are critical for studying emerging IDs, permitting wide-ranging epidemiological and laboratory analyses of suspected pathogens (43). Molecular methods are most appropriate for agents difficult to culture, isolate, or characterize in a timely manner by the more conventional methods, but are playing an increasingly important role in studies of emerging diseases (42-44).

For immunoassays, the most critical component will likely remain the antibody. Ultimately, improvements in the affinity, specificity, and mass production of antibodies will dictate the success or failure of a given immunoassay technology. For nucleic acid-based assays, the most critical component will likely remain the availability of genomic sequence data from pathogenic organisms and more benign, but related, organisms. Advances in reagents and assay formats are being matched by improvements in complementary technology, ranging from automated analyzers and microarrays that facilitate the analysis of large numbers of samples, to self-contained miniaturized devices that enable an assay to be performed at the point of care or in a field setting.

Currently, well-established molecular methods such as PCR and ELISA are widely used for the analysis of pathogenic agents. Waiting in the wings are array-based assays, which will allow a range of phenotypic and genotypic traits to be rapidly and simultaneously determined. Application of these latter technologies to the study of BW/ID agents, when coupled with the increasing depth of genomic sequence data, holds incredible promise. For example, sequence-based identification and strain typing, together with the development of tools to probe for thousands of markers, will allow detailed strain fingerprinting, which, in turn, will greatly facilitate disease management and control (28). Further extension of these technologies to permit near instantaneous detection of potential pathogens, combined with simultaneous prediction of drug resistance profiles, virulence factors, and pathogenicity islands, would revolutionize clinical microbiology and the management of ID. At this point in time, however, the primary role of such arrays is for research, as fabrication costs decrease, system robustness and reliability improve, and fully validated sets of arrays are developed, it is inevitable they will be used for pathogen detection and characterization (45).

During this period of increasing access to microbial sequence data, parallel advances have occurred in techniques that allow the large-scale study of the entire genetic complement of microorganisms (46). In the near future, these approaches will enable researchers to unravel further the complexity of microbial pathogenesis and identify new virulence determinants. Many of these have proved to be suitable targets for development as diagnostic reagents, antimicrobial agents, and vaccine candidates. Although it is difficult to predict the full impact that this volume of information will have on the practice of microbiology and immunology, it will give rise to ever faster and more robust detection and identification methods for laboratory and field use. Sensitive and specific detection of BW and ID agents has improved by several orders of magnitude over the past 30 years. If recent scientific progress is a fair indicator, the future promises continued improvements through an everincreasing array of applications.

\section{REFERENCES}

1.Kortepeter, M.G. and G.W. Parker. 1999. Potential biological weapons threats. Emerg. Infect. Dis. 5:523-527.

2.Nichol, S.T., J. Arikawa, and Y. Kawaoka. 2000. Emerging viral diseases. Proc. Natl. Acad. Sci. USA 97:12411-12412.

3.Rotz, L.D., A.S. Khan, S.R. Lillibridge, S.M. Ostroff, and J.M. Hughes. 2002. Public health assessment of potential biological terrorism agents. Emerg. Infect. Dis. 8:225-230.

4.Vorobjev, A.A., B.L. Cherkassey, A.V. Stepanow, A.A. Kyuregyan, and Y.M. Fjedorov. 1997. Key problems of controlling especially dangerous infections. In Proceedings of the International Symporium of Severe Infectious Disease: Epidemiology, Express-Diagnostics and Prevention. State Scientific Institution, Volgo-Vyatsky Center of Applied Biotechnology. Kirov, Russia.

5.Cieslak, T.J., J.R. Rowe, M.G. Kortepeter, J.M. Madsen, J. Newmark, G.W. Christopher, R.C. Culpepper, and E.M. Eitzen, Jr. 2000. A fieldexpedient algorithmic approach to the clinical management of chemical and biological casualties. Mil. Med. 165:659-662.

6.Franz, D.R., P.B. Jahrling, A.M. Friedlander, D.J. McClain, D.L. Hoover, W.R. Bryne, J.A. Pavlin, G.W. Christopher, and E.M. Eitzen, Jr. 1997. Clinical recognition and management of patients exposed to biological warfare agents. JAMA 278:399-411.

7.Franz, D.R., P.B. Jahrling, D.J. McClain, D.L. Hoover, W.R. Byrne, J.A. Pavlin, G.W. Christopher, T.J. Cieslak, et al. 2001. Clinical recognition and management of patients exposed to biological warfare agents. Clin. Lab. Med. 21:435-473.

8.National Research Council. 1999. Chemical and Biological Terrorism: Research and Development to Improve Civilian Medical Response, p. 6596. National Academy Press, Washington, DC.

9.Snyder, J.W. 2000. Cumitech 33: Biological Agents Associated with Bio- 
terrorism. ASM Press, Washington, DC

10.Murray, P.R. 2003. Manual of Clinical Microbiology. ASM Press, Washington, DC.

11.Hail, A.S., C.A. Rossi, G.V. Ludwig, B.E. Ivins, R.F. Tammariello, and E.A. Henchal. 1999. Comparison of noninvasive sampling sites for early detection of Bacillus anthracis spores from rhesus monkeys after aerosol exposure. Mil. Med. 164:833-837.

12.Gilchrist, M.J. 2000. A national laboratory network for bioterrorism: evolution from a prototype network of laboratories performing routine surveillance. Mil. Med. 165:28-31.

13.Henchal, E.A., J.D. Teska, and J.W. Ezzell. 2000. Responding to biological terrorism: a role for the clinical laboratory. Clin. Lab. News 26: 14-18.

14.Ivnitski, D., D.J. O'Neil, A. Gattuso, R. Schlicht, M. Calidonna, and R. Fisher. 2003. Nucleic acid approaches for detection and identification of biological warfare and infectious disease agents. BioTechniques 35 : 862-869.

15.Garcia-de-Lomas, J. and D. Navarro. 1997. New directions in diagnostics. Pediatr. Infect. Dis. J. 16:S43-S48.

16.Whelen, A.C. and D.H. Persing. 1996. The role of nucleic acid amplification and detection in the clinical microbiology laboratory. Ann. Rev. Microbiol. 50:349-373.

17.Wu, X., G. Cheng, B. Di, A. Yin, Y. He, M. Wang, X. Zhou, L. He, et al. 2003. Establishment of a fluorescent polymerase chain reaction method for the detection of the SARS-associated coronavirus and its clinical application. Chin. Med. J. (Engl) 116:988-990.

18.McIntosh, K. 2003. The SARS coronavirus: rapid diagnostics in the limelight. Clin. Chem. 49:845-846.

19.Hoffmaster, A.R., R.F. Meyer, M.D. Bowen, C.K. Marston, R.S. Weyant, K. Thurman, S.L. Messenger, E.E. Minor, et al. 2002. Evaluation and validation of a real-time polymerase chain reaction assay for rapid identification of Bacillus anthracis. Emerg. Infect. Dis. 8:1178-1182.

20.Higgins, J.A., M. Cooper, L. Schroeder-Tucker, S. Black, D. Miller, J.S. Karns, E. Manthey, R. Breeze, and M.L. Perdue. 2003. A field investigation of Bacillus anthracis contamination of U.S. Department of Agriculture and other Washington, D.C., buildings during the anthrax attack of October 2001. Appl. Environ. Microbiol. 69:593-599.

21.Heller, M.B., M.L. Bunning, M.E. France, D.M. Niemeyer, L. Peruski, T. Naimi, P.M. Talboy, P.H. Murray, et al. 2002. Laboratory response to anthrax bioterrorism, New York City, 2001. Emerg. Infect. Dis. 8:1096-1102.

22.Henchal, E.A. and M.S. Ibrahim. 2000. Evaluation of polymerase chain reaction assays for identifying biological agents, p. 239-249. In M.A. Bartoszcze (Ed.), Rapid Methods for Analysis of Biological Materials in the Environment. Kluwer Academic Publishers, Amsterdam, Netherlands.

23.Livak, K.J., S.J. Flood, J. Marmaro, W. Giusti, and K. Deetz. 1995. Oligonucleotides with fluorescent dyes at opposite ends provide a quenched probe system useful for detecting PCR product and nucleic acid hybridization. PCR Methods Appl. 4:357-362.

24.Livak, K.J., J. Marmaro, and J.A. Todd. 1995. Towards fully automated genome-wide polymorphism screening. Nat. Genet. 9:341-342.

25.Henchal, E.A., J.D. Teska, G.V. Ludwig, D.R. Shoemaker, and J.W. Ezzell. 2001. Current laboratory methods for biological threat agent identification. Clin. Lab. Med. 21:661-678.

26.Kato-Maeda, M., Q. Gao, and P.M. Small. 2001. Microarray analysis of pathogens and their interaction with hosts. Cell. Microbiol. 3:713-719.

27.Vernet, G. 2002. DNA-chip technology and infectious diseases. Virus Res. 82:65-71.

28.Gilbert, G.L. 2002. Molecular diagnostics in infectious diseases and public health microbiology: cottage industry to postgenomics. Trends Mol. Med. 8:280-287.

29.Andreotti, P., G.V. Ludwig, A.H. Peruski, J. Tuite, S. Morse, and L.F. Peruski, Jr. 2003. Immunoassay of infectious agents. BioTechniques 35: 850-859.

30.Peruski, A.H. and L.F. Peruski, Jr. 2003. Immunological methods for detection and identification of infectious disease and biological warfare agents. Clin. Diagn. Lab. Immunol. 10:506-513.

31.Baselt, D.R., G.U. Lee, M. Natesan, S.W. Metzger, P.E. Sheehan, and R.J. Colton. 1998. A biosensor based on magnetoresistance technology. Biosens. Bioelectron. 13:731-739.

32.Horton, J.K., S. Swinburne, and M.J. O'Sullivan. 1991. A novel, rapid, single-step immunochromatographic procedure for the detection of mouse immunoglobulin. J. Immunol. Methods 140:131-134.

33.Kriz, K., J. Gehrke, and D. Kriz. 1998. Advancements toward magneto immunoassays. Biosens. Bioelectron. 13:817-823.

34.Richardson, J., P. Hawkins, and R. Luxton. 2001. The use of coated paramagnetic particles as a physical label in a magneto-immunoassay. Biosens. Bioelectron. 16:989-993.

35.Katti, M.K. 2001. Are enzyme-linked immunosorbent assay and immunoblot assay independent in immunodiagnosis of infectious diseases? Clin. Infect. Dis 32:1114

36.Payne, W.J., Jr., D.L. Marshall, R.K. Shockley, and W.J. Martin. 1988. Clinical laboratory applications of monoclonal antibodies. Clin. Microbiol. Rev. 1:313-329.

37.Wright, P.F., E. Nilsson, E.M. Van Rooij, M. Lelenta, and M.H. Jeggo. 1993. Standardisation and validation of enzyme-linked immunosorbent assay techniques for the detection of antibody in infectious disease diagnosis. Rev. Sci. Tech. 12:435-450.

38.Peruski, A.H., L.H. Johnson III, and L.F. Peruski, Jr. 2002. Rapid and sensitive detection of biological warfare agents using time-resolved fluorescence assays. J. Immunol. Methods 263:35-41.

39.Diamandis, E.P. and T.K. Christopoulos. 1991. Time-resolved immunofluorometric detection of antigens separated by high-performance liquid chromatography and coated to polystyrene. BioTechniques 10:646-648.

40.Ieven, M. 2000. Molecular methods in the diagnostic microbiology laboratory: when to start and where to stop? Verh. K. Acad. Geneeskd. Belg. 62:15-29.

41.Smith, D.R., C.A. Rossi, T.M. Kijek, E.A. Henchal, and G.V. Ludwig. 2001. Comparison of dissociation-enhanced lanthanide fluorescent immunoassays to enzyme-linked immunosorbent assays for detection of staphylococcal enterotoxin B, Yersinia pestis-specific F1 antigen, and Venezuelan equine encephalitis virus. Clin. Diagn. Lab. Immunol. 8: 1070-1075

42.Dumler, J.S. and A. Valsamakis. 1999. Molecular diagnostics for existing and emerging infections. Complementary tools for a new era of clinical microbiology. Am. J. Clin. Pathol. 112:S33-S39.

43.Houpikian, P. and D. Raoult. 2002. Traditional and molecular techniques for the study of emerging bacterial diseases: one laboratory's perspective. Emerg. Infect. Dis. 8:122-131.

44.Pfaller, M.A. 2001. Molecular approaches to diagnosing and managing infectious diseases: practicality and costs. Emerg. Infect. Dis 7:312-318.

45.Anthony, R.M., T.J. Brown, and G.L. French. 2001. DNA array technology and diagnostic microbiology. Expert. Rev. Mol. Diagn. 1:30-38.

46.Jenks, P.J. 1998. Sequencing microbial genomes-what will it do for microbiology? J. Med. Microbiol. 47:375-382.

\section{Address correspondence to:}

Leonard F. Peruski, Jr. or Anne Harwood Peruski

Department of Microbiology and Immunology

Northwest Center for Medical Education

Indiana University School of Medicine

3400 Broadway

Gary, IN 46408, USA

e-mail:lperuski@iun.edu oraperuski@iun.edu 